\title{
WILEY-VCH
}

This is the peer reviewed version of the following article: Shaohua Liu, Faxing Wang, Renhao Dong, Tao Zhang, Jian Zhang, Zhikun Zheng, Yiyong Mai, and Xinliang Feng. Soft-Template Construction of 3D Macroporous Polypyrrole Scaffolds. Small 2017, 13, 1604099, which has been published in final form at https://doi.org/10.1002/smll.201604099. This article may be used for non-commercial purposes in accordance with Wiley Terms and Conditions for Self-Archiving. 
DOI: $10.1002 /(($ please add manuscript number $))$

Article type: Communication

\section{Soft-Template Construction of 3D Macroporous Polypyrrole Scaffolds}

Shaohua Liu, Faxing Wang, Renhao Dong, Tao Zhang, Jian Zhang, Zhikun Zheng, Yiyong Mai, and Xinliang Feng*

Dr. S. Liu, Mr. F. Wang, Dr. R. Dong, Dr. J. Zhang, Dr. Z. Zheng, Prof. X. Feng Center for Advancing Electronics Dresden (cfaed) \& Department of Chemistry and Food Chemistry, Technische Universität Dresden, 01062 Dresden, Germany

E-mail: xinliang.feng@tu-dresden.de

Prof. Y. Mai

School of Chemistry and Chemical Engineering, Shanghai Key Laboratory of Electrical Insulation and Thermal Ageing, Shanghai Jiao Tong University, 800 Dongchuan Road, 200240, Shanghai, P. R. China

Keywords: three-dimensional (3D) • macroporous $\bullet$ self-assembly $\bullet$ conducting polymer • nanosheets

Inspired by the unique structure, intriguing electronic and physical properties of graphene, ${ }^{[1-4]}$ increasing attentions have been devoted to exploring novel two-dimensional (2D) nanomaterials. In the last decade, a series of $2 \mathrm{D}$ nanomaterials, including inorganic ${ }^{[1,2,5-9]}$ and organic/polymeric nanosheets, ${ }^{[10-14]}$ have been prepared by top-down mechanical cleavage, chemical and electrochemical exfoliation ${ }^{[1,2,5-9,15-18]}$ as well as bottom-up vapor-liquid-solid and vapor-solid growth techniques. ${ }^{[11-14]}$ However, those nanosheets with ultrathin features usually suffer from the re-stacking and re-aggregation driven by high surface free energy or interlayer van der Waals forces, which would remarkably hamper their utilization in the areas including energy storage, catalysis and chemical sensing, etc..

One promising strategy to alleviate the strong aggregation of ultrathin nanosheets is to arrange them into 3D aerogels. Those 3D architectures composed of 2D ultrathin nanosheets could simultaneously achieve both advantages of nanosheet structure and macroscopic features. ${ }^{[19-24]}$ Meanwhile, 3D aerogels with interconnected macropores would provide interpenetrated channels and high specific surface areas, which are essential for the access of 


\section{WILEY-VCH}

electrolyte and the fast diffusion of ions when serving as electrodes for energy storage devices. ${ }^{[19-26]}$ To date, various 3D macroporous materials and their composites have been built up through the well-established strategies, such as self-assembly, template-directed synthesis or direct growth on 3D foam templates. ${ }^{[19-26]}$ However, the building blocks for those 3D materials have been limited so far in a narrow range of 2D nanosheets based on inorganic metal oxides, nitrides, sulfides and graphene (oxides). ${ }^{[19-27]}$ Although conducting polymers with conjugated backbones have been extensively explored for the utilization as energy storage materials due to their relatively low cost, easy fabrication, high electrical conductivity and excellent electrochemical behavior, direct bottom-up construction of 3D macroporous materials employing ultrathin conducting polymer nanosheets has remained unrealized. ${ }^{[19-33]}$

Herein, we demonstrate a bottom-up approach towards 3D hierarchical macroporous conducting polymer aerogels via soft template-directed synthesis and self-assembly of ultrathin polypyrrole (PPy) nanosheets in solution, involving that perfluorocarboxylic acids (PFCA) amphiphiles self-assemble into lamellas with a bilayer structure in ethanol/ $\mathrm{H}_{2} \mathrm{O}$ solution, which guide the polymerization of pyrrole monomers into ultrathin PPy nanosheets. The further polymerization results in the cross-linking of the adjacent PPy nanosheets and establishment of the 3D macroporous PPy gels (PPGs). Upon template removal of PFCA by extraction with ethanol and subsequent freeze-drying process, 3D PPGs are achieved with ultrathin building units (thicknesses of $10 \sim 16 \mathrm{~nm}$ ), macroporous structure $(1 \sim 2 \mu \mathrm{m})$ and large specific surface areas $\left(59 \mathrm{~m}^{2} \mathrm{~g}^{-1}\right)$. Serving as cathode materials for Na-ion batteries, the resulting 3D macroporous PPGs exhibit a high Na-ion storage capacity up to $106 \mathrm{~mA} \mathrm{~h} \mathrm{~g}$ at $80 \mathrm{~mA} \mathrm{~g}^{-1}$, satisfactory rate capability and excellent cycling stability with a capacity retention of about $95 \%$ even after 200 cycles at a high rate of $150 \mathrm{~mA} \mathrm{~g}^{-1}$. Such performance is superior to those of the most recently reported cathode materials for Na-ion batteries (usually less than $100 \mathrm{mAh} \mathrm{\textrm {g } ^ { - }}$ $\left.{ }^{1}\right) \cdot{ }^{[34-36]}$ 


\section{WILEY-VCH}
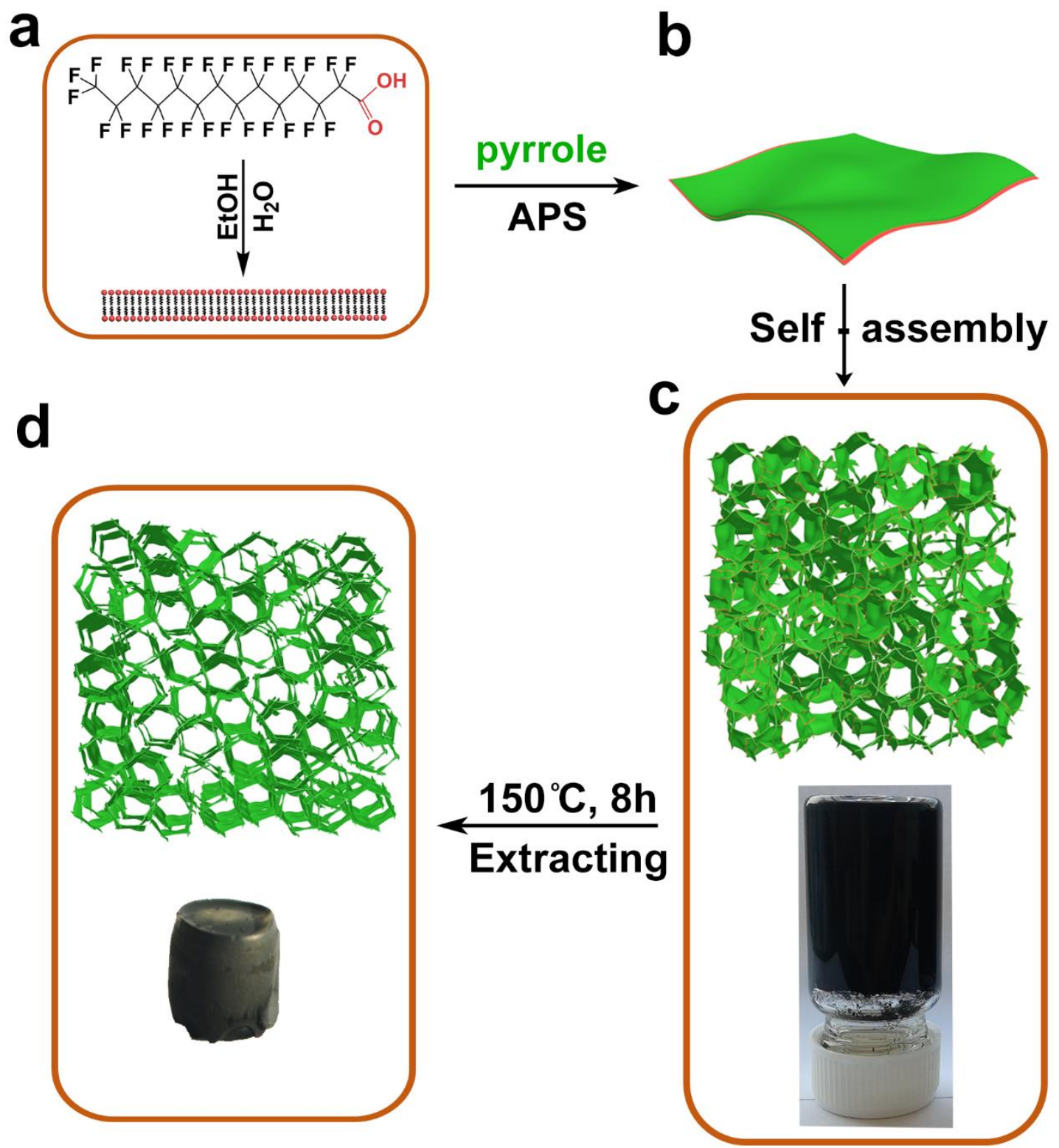

Figure 1. Schematic illustration for the construction of 3D PPGs. a) The formation of ultrathin lamellae of PFTA in mixed ethanol/ $\mathrm{H}_{2} \mathrm{O}$ solvents at room temperature. $\mathbf{b}$, c) The formation of 2D PPy nanosheets and further cross-link into 3D PPy/PFTA hydrogel, after adding ammonium persulfate (APS). d) The final 3D PPy aerogels prepared by solidification, extraction and freeze-drying.

As illustrated in Figure 1, in order to construct 3D nanosheet-based macroporous PPGs, perfluorotetradecanoic acid (PFTA) amphiphile was chosen as the 2D template. Our recent report demonstrated that PFTA can self-organize into well-defined lamellae bilayer in mixed ethanol/ $\mathrm{H}_{2} \mathrm{O}(\mathrm{v} / \mathrm{v}=1: 5)$ solvents at room temperature (Figure 1a). ${ }^{[37-39]}$ Because of cryogenic transmission electron microscopy (cryo-TEM) allows the direct observation of specimens at cryogenic temperatures (generally liquid-nitrogen temperatures), showing them in their native 


\section{WILEY-VCH}

environment, here we used cryo-TEM to in-situ observe the morphology and structure of surfactant micelles of PFCA. Figure S1 in Supporting Information (SI) reveals the presence of the transparent nanosheets with the size up to $3 \sim 4 \mu \mathrm{m}$, suggestive of the formation of PFTA lamellae in $\mathrm{EtOH} / \mathrm{H}_{2} \mathrm{O}$ mixture solution. After adding pyrrole molecules into the PFTA lamella solution, they could be adsorbed on both surfaces of the PFTA lamellae through hydrogen bonding between the carboxylic acid groups of PFTA and the $-\mathrm{NH}$ moieties of pyrrole. ${ }^{[40]}$ Subsequently, ammonium persulfate (APS) was added, which triggered the polymerization of pyrrole monomers on the PFTA lamellae, thereby forming 2D PPy nanosheets (Figure 1b). The further growth of 2D PPy nanosheets led to the formation of cross-linked polymeric frameworks upon a heat treatment at $150{ }^{\circ} \mathrm{C}$ for $8 \mathrm{~h}$ (Figure 1c). After removal of the PFTA template by extraction with $1 \mathrm{M} \mathrm{HCl}$ solution in ethanol and then freeze-drying process, free-standing nanosheet-based 3D macroporous PPGs were obtained (Figure 1d).
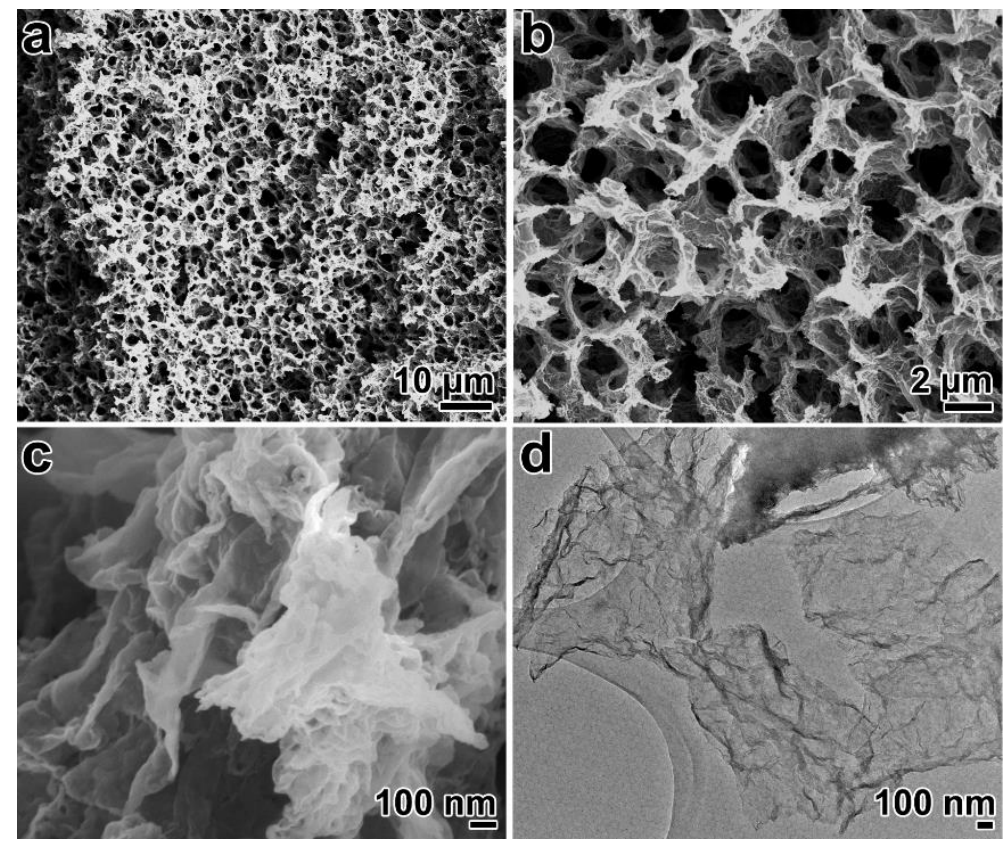

Figure 2. The microstructure of the solidified 3D PPGs at $150{ }^{\circ} \mathrm{C}$. (a-c) SEM images with different magnifications, and (d) TEM images of the solidified 3D PPGs.

The Fourier-transform infrared (FT-IR) spectra of the 3D PPGs (Figure S2 in SI) suggest the complete removal of PFTA by the disappearance of the characteristic peaks of the carboxylic acid group, $\mathrm{C}-\mathrm{F}$ bonds and the alkyl chain of PFTA. Meanwhile, the appearance of 


\section{WILEY-VCH}

the characteristic peaks of $\mathrm{C}=\mathrm{N}\left(1685 \mathrm{~cm}^{-1}\right)$ and $\mathrm{C}-\mathrm{N}\left(1315 \mathrm{~cm}^{-1}\right)$ manifests the formation of PPy. Additionally, a series of peaks at 921, 976, 1043, 1322 and $1564 \mathrm{~cm}^{-1}$, corresponding to a reduced PPy, ${ }^{[41]}$ have been found in Raman spectra of the 3D PPGs that can also verifies the presence of PPy (Figure S3 in SI).

The microstructure of the as-made 3D PPGs were investigated by scanning electron microscopy (SEM) and transmission electron microscopy (TEM). As shown in Figure S4 and 5 (SI), the as-made free-standing 3D PPGs possess interconnected macropores of dimensions in the range of $2 \sim 3 \mu \mathrm{m}$. Upon heat treatment at $150{ }^{\circ} \mathrm{C}$ for $8 \mathrm{~h}$, the as-made 3D PPGs would become more stable due to the further cross-linking of PPy. Compared to their as-synthesized 3D counterparts, the interconnected macropores of the additional thermally treated 3D PPGs became more uniform and displayed smaller pore size $(\sim 2 \mu \mathrm{m})$. Figure 2 clearly revealed that the 3D macroporous PPGs were composed of the ultrathin PPy nanosheets. The partial coalescence and overlapping of 2D flexible PPy nanosheets might originate from the crosslinking of the PPy nanosheets, which would efficiently support the 3D PPGs with interconnected macroporous architecture.

As the building units of the 3D PPGs, the intermediate ultrathin PPy nanosheets can be directly isolated from the as-synthesized 3D PPGs before solidification into 3D aerogels by separation and removal of PFTA template (see Experiment Section). The direct microscopy observation confirmed their 2D nanosheet morphology with the typical lateral sizes up to several micrometers (Figure 3a, b and Figure S6). Furthermore, atomic force microscopy (AFM) analysis validated that these PPy nanosheets possessed an average thickness of $\sim 10 \mathrm{~nm}$ (Figure S7 in SI). However, despite of the same carbon chains with PFTA, the use of myristic acid as amphiphile only led to the formation of spherical-like PPy nanoparticles that quickly precipitated from solution, rather than forming stable 3D gels (Figure S8 and S9 in SI). This result strongly suggests that the PFTA bilayer template plays the crucial role for the directed growth of pyrrole monomers into 2D PPy nanosheets and the subsequent self-assembly into 3D 


\section{WILEY-VCH}

PPGs. Compared with myristic acid, PFTA molecules possess higher interface activity and lower van der Waals force, which can facilitate their self-assembly into stable lamellae with a bilayer structure that results in a remarkable decrease of the surface tension of solution. ${ }^{[42]}$

The porous feature of 3D PPGs is confirmed by Brunauer-Emmett-Teller (BET) measurements. The $\mathrm{N}_{2}$ sorption isotherms (Figure 3c) show a typical II hysteresis loop at a relative pressure of $0.40 \sim 0.95$, a characteristic of pores with macropore/mesopore sizes. ${ }^{[43]}$ Apart from the macropores of $2 \mu \mathrm{m}$ estimated from the SEM images, Barrett-Joyner-Halenda (BJH) results also reveal the existence of mesopores with sizes in the range of $2 \sim 15 \mathrm{~nm}$ for the 3D PPGs (Figure S10 in SI). The BET surface area and pore volume of the 3D PPGs are calculated to be $54 \mathrm{~m}^{2} \mathrm{~g}^{-1}$ and $0.12 \mathrm{~cm}^{3} \mathrm{~g}^{-1}$ (Table $\mathrm{S} 1$ in SI), respectively, which surpass many of the previously reported PPy materials (Table S2 in SI).
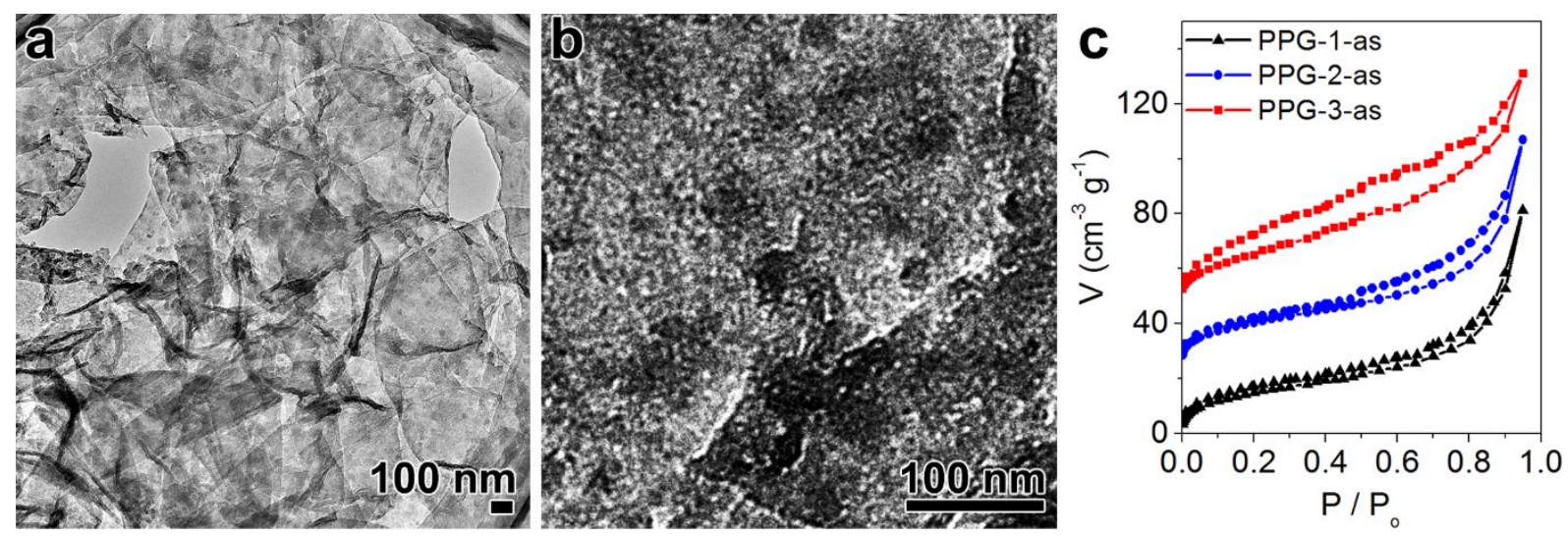

Figure 3. The morphology and microstructure of the PPy nanosheet and their derived 3D PPGs synthesized with PFTA as the templates. (a, b) TEM images of the PPy nanosheet of the asmade 3D PPGs. (c) Nitrogen adsorption-desorption isotherm of the as-made 3D PPGs synthesized with different amounts of pyrrole precursor, and (d) the calcined samples at $900{ }^{\circ} \mathrm{C}$ under inert atmosphere. For the as-made 3D PPG-2 and 3, the isotherms are offset vertically by 25 and $50 \mathrm{~cm}^{3} \mathrm{~g}^{-1}$ STP, respectively; similarly, the isotherms of the calcined 3D PPG-2 and 3 samples are offset vertically by 250 and $400 \mathrm{~cm}^{3} \mathrm{~g}^{-1} \mathrm{STP}$, respectively.

The thickness of PPy nanosheets, as the building units for 3D PPGs, can be adjusted by varying the amounts of pyrrole monomer during the PFTA-directed polymerization (the mole ratios of PFTA to pyrrole can be 1.56, 1.18 and 0.94, named as PPG-1, 2 and 3, respectively.) Figure S5 shows that all of the PPG samples possessed 3D macroporous framework structure. 


\section{WILEY-VCH}

BJH calculations disclosed that PPG-1, 2 and 3 had a similar surface area $\left(50 \sim 60 \mathrm{~m}^{2} \mathrm{~g}^{-1}\right)$ and pore volume $\left(0.08 \sim 0.12 \mathrm{~cm}^{3} \mathrm{~g}^{-1}\right)$. All of the 3D PPGs exhibited enlarged surface areas compared with that of the controlled PPy sample (PPy-blank, $25 \mathrm{~m}^{2} \mathrm{~g}^{-1}$, Figure S8 in SI). Notably, further calcination of 3D PPGs under inert atmosphere at $900{ }^{\circ} \mathrm{C}$ led to the transformation into 3D macroporous carbon aerogels with a remarkable increase of the surface areas, reaching up to $\sim 502 \mathrm{~m}^{2} \mathrm{~g}^{-1}$ for the $3 \mathrm{D}$ PPG-1 sample (Figure S10 and 11 in SI).
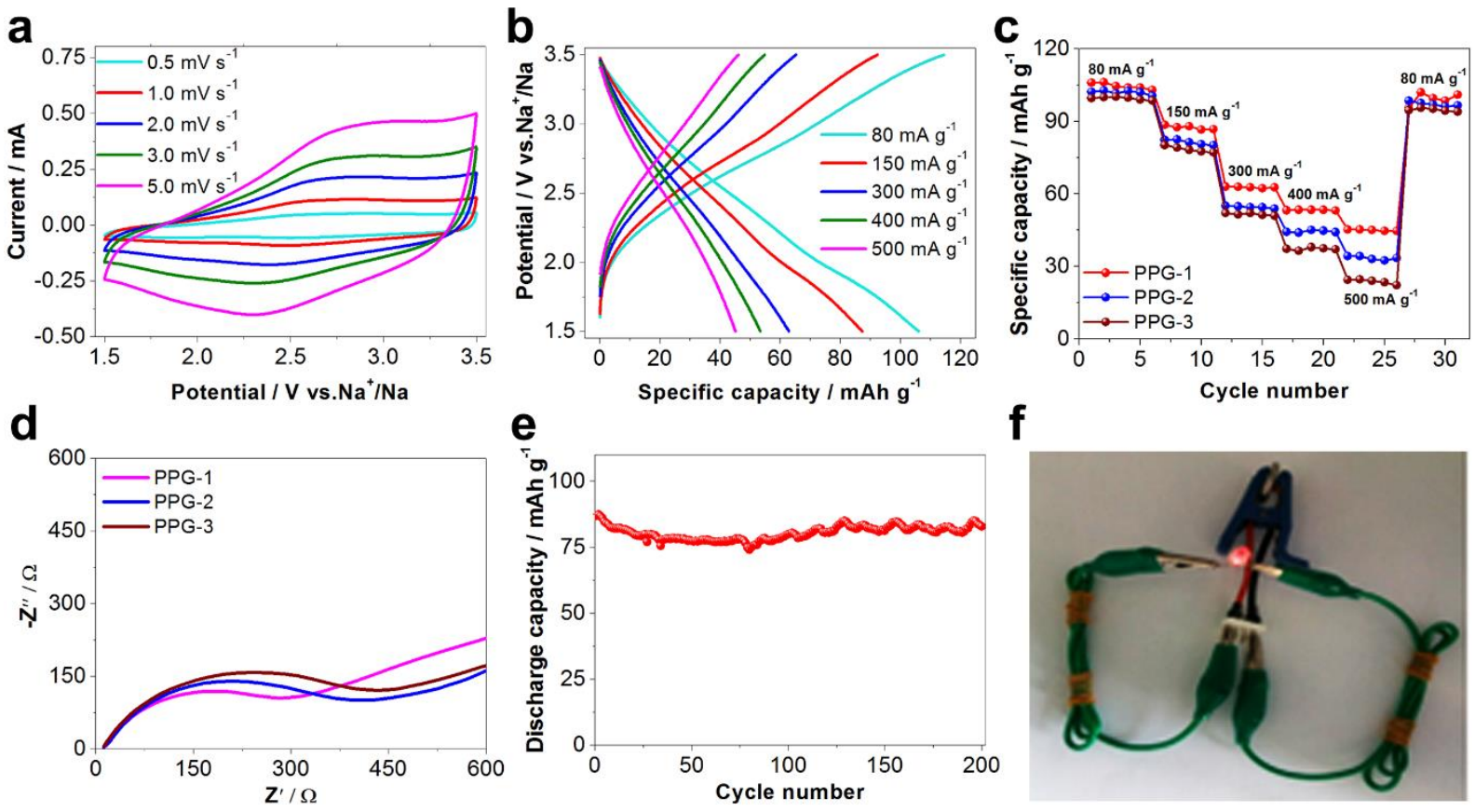

Figure 4. Electrochemical properties of 3D PPG electrodes for Na ion battery. a) CV curves of the 3D PPG-1 at different scan rates. b) Its galvanostatic charge/discharge curves at different current densities. c) Its rate capabilities. d) The Nyquist plots of 3D PPG-1, PPG-2 and PPG-3. e) The cycling behavior of 3D PPG-1 at a current density of $150 \mathrm{~mA} \mathrm{~g}$. $\mathrm{f}$ ) An optical photograph showing a lighted red LED, which was powered by using the 3D PPG-1 based Na ion battery.

Profiting from the hierarchical porous structure, ultrathin nanosheets and large specific surface areas, the resultant 3D PPGs hold promise as electrode materials for energy storage devices. The redox reaction in PPy usually involves p-doping/de-doping electrolyte anions into/from the polymer chains, which is not influenced by the cation species, rendering PPy as a suitable cathode material for Na-based energy storage system. ${ }^{[44-49]}$ Thereby, we investigated the electrochemical performance of the 3D PPGs in $1 \mathrm{M} \mathrm{NaPF}_{6} / \mathrm{DEGDME}$ (diethylene glycol 


\section{WILEY-VCH}

dimethyl ether) electrolyte. The cyclic voltammetry (CV) curves of the 3D PPGs showed the redox peaks at $2.4 \mathrm{~V}$ during the reduction process and at $2.6 \mathrm{~V}$ during the oxidation process in the initial cycle at a low scan rate (Figure 4a). Although the peak separation increased upon increasing the scan rate, 3D PPG-1 electrode still retained the well-defined shape even after the scan rate increases to $5 \mathrm{mV} \mathrm{s}^{-1}$.

Next, we examined the galvanostatic charge/discharge behavior of the 3D PPGs based Naion batteries at various current densities (Figure 4b). The 3D PPGs-1 electrode delivered a reversible discharge capacity of $106 \mathrm{~mA} \mathrm{~h} \mathrm{~g}^{-1}$ at $80 \mathrm{~mA} \mathrm{~g}^{-1}$, which is substantially higher than many PPy-based cathode materials of various morphologies, such as nanoparticles and hollow spheres. ${ }^{[44-46,48]}$ Additionally, the 3D PPG-1 electrode delivered a better rate capability $(87,63$, 55 and $45 \mathrm{~mA} \mathrm{~h} \mathrm{~g}^{-1}$ at current densities of $150,300,400$ and $500 \mathrm{~mA} \mathrm{~g}^{-1}$, respectively) than those of 3D PPG-2 and 3D PPG-3 electrodes (Figure 4c). This result suggests that the relatively thinner nanosheets of 3D PPG-1 electrode should provide more electrochemical active sites and electrolyte/electrode contact interfaces for ion storage, thus leading to a high specific capacity. As an eco-friendly and metal-free electrode material, the 3D PPG-based electrodes exhibited a competitive performance compared with those of the recently reported cathode materials for Na-ion batteries, such as $\mathrm{Na}_{0.6} \mathrm{Cr}_{0.6} \mathrm{Ti}_{0.4} \mathrm{O}_{2}\left(<75 \mathrm{mAh} \mathrm{g}^{-1}\right.$ at $\left.7.6 \mathrm{~mA} \mathrm{~g}{ }^{-1}\right),{ }^{[35]} \mathrm{Na}_{2} \mathrm{Fe}_{2}\left(\mathrm{SO}_{4}\right)_{3}(100$ mAh g ${ }^{-1}$, current density less than $\left.30 \mathrm{~mA} \mathrm{~g}^{-1}\right)^{[50]}$ and $\mathrm{Na}_{2} \mathrm{MnP}_{2} \mathrm{O}_{7}\left(90 \mathrm{mAh} \mathrm{g}^{-1}\right.$, current density less than $\left.10 \mathrm{~mA} \mathrm{~g}^{-1}\right) \cdot{ }^{[36]}$ Such the ultrathin nanosheets of the 3D PPG-based electrodes would shorten the ion diffusion and electron transport path, thereby yielding remarkable rate capability. ${ }^{[40]}$ By comparison, the controlled PPy nanoparticles synthesized without any templates show lower special capability $\left(<20 \mathrm{mAh} \mathrm{g}^{-1}\right.$ at $\left.80 \mathrm{~mA} \mathrm{~g}^{-1}\right)$, rate capability and poor cycle stability (Figure S12 in SI). This result indicates the significant contribution of our unique 2D nanosheet-based macroporous architectures to the $\mathrm{Na}$ ion storage.

Additionally, electrochemical impedance spectroscopy (EIS) studies of the 3D PPG cathodes (Figure 4d) showed the similar EIS profiles, with a semicircle in the high-frequency 


\section{WILEY-VCH}

region and a straight line in the low-frequency region. The charge-transfer resistance $\left(\mathrm{R}_{\mathrm{ct}}\right)$ is estimated to be $290 \mathrm{ohm}$ for the 3D PPG-1 electrode (Fig. 4d), which is noticeably lower than those for the PPG-2 (380 ohm) and PPG-3 (430 ohm) electrodes. Notably, the polymer cathode maintained its initial capacity of $87 \mathrm{~mA} \mathrm{~h} \mathrm{~g}^{-1}$ and capacity retention of about $95 \%$ even after 200 cycles at a high rate of $150 \mathrm{~mA} \mathrm{~g}^{-1}$ (Figure 4e). This cycling stability of the 3D PPy-1 nanosheets is superior to the those of PPy hollow nanospheres and sulfonated polyaniline for Na-ion batteries. ${ }^{[44-49]}$ Moreover, the 3D PPG-based electrodes at $80 \mathrm{~mA} \mathrm{~g}^{-1}$ could readily power a commercial red LED (1.7-2.3 V) (Figure $4 \mathrm{f}$ ), suggesting a great potential of 3D PPGs in Na-ion batteries.

In conclusion, we demonstrated a novel bottom-up route towards the fabrication of 3D PPy nanosheet-based macroporous aerogels via soft template-directed self-assembly of polymer precursor. The lamellae formed by amphiphilic surfactant of perfluorocarboxylic acid in solution served as 2D soft templates and guided the polymerization of pyrrole monomer into 2D ultrathin PPy nanosheets and then self-assemble into 3D PPGs. The resultant 3D PPGs possessed thickness-adjusted nanosheets as the building units, macroporous structure and high specific surface areas. Such unique 3D structure endows them with a high storage capacity, satisfactory rate capability and excellent cyclability when serving as cathode materials for Naion batteries. This study provides new insights for construction of nanosheet-based 3D macroporous materials directly from molecular precursors towards diverse applications.

\section{Experimental Section}

Synthesis of $3 D$ macroporous polypyrrole gels (PPGs). Typically, $0.05 \mathrm{~g}$ of perfluorotetradecanoic acid (PFTA) was dissolved in $2 \mathrm{~mL}$ ethanol and $10 \mathrm{~mL} \mathrm{H}_{2} \mathrm{O}$ to generate the micellar aggregation. After stirring, $62 \mu \mathrm{L}$ of pyrrole was added to the above solution. After adding $0.18 \mathrm{~g}$ ammonium persulfate (APS) and continuous stirring for $10 \mathrm{~min}$ and staying for $1 \mathrm{~h}$, the solution turned eventually dark and gelated. The above solution was transferred into 


\section{WILEY-VCH}

Teflon lined stainless steel autoclaves and maintained at $150{ }^{\circ} \mathrm{C}$ for $8 \mathrm{~h}$. PFTA template and the other excess ions can be removed by extraction with $1 \mathrm{M} \mathrm{HCl} / \mathrm{ethanol}$ solution and rinse by deionized water (DI water). Final sample of 3D macroporous PPy aerogel was obtained by freeze-drying. The intermediate ultrathin PPy nanosheets can be directly isolated from the assynthesized 3D PPGs before solidification into 3D aerogels by separation and removal of PFTA template, including the repeated ultrasound desperation into EtOH, washing with EtOH and $\mathrm{H}_{2} \mathrm{O}$, centrifugation separation as well as subsequent exaction using $\mathrm{HCl} / \mathrm{EtOH}$ solution, etc. Electrochemical measurements of 3D PPGs as cathode for Na-ion batteries. The as-prepared samples were each mixed with acetylene black and polyvinylidene difluoride (PVDF) in a weight ratio of $7: 1.5: 1.5$, respectively, with the help of N-methyl-2-pyrrolidone (NMP). Then the mixture was coated on aluminum foil using a doctor-blade technique. After that, the coated foils were dried and punched into circular pieces $(\mathrm{d}=11 \mathrm{~mm})$, which were further dried at 80 ${ }^{\circ} \mathrm{C}$ for $12 \mathrm{~h}$ under vacuum. CR2032-type coin cells were assembled by sandwiching a porous polypropylene separator between the working electrode and $\mathrm{Na}$ metal foil in a high-purity argon-filled glove box with the contents of oxygen and water below $0.1 \mathrm{ppm}$. The electrolyte was $1.0 \mathrm{M} \mathrm{NaPF}_{6}$ in the diethylene glycol dimethyl ether (DEGDME). Cyclic voltammogram (CV) measurements were performed on a $\mathrm{CHI} 650 \mathrm{E}$ electrochemical workstation (Chenhua Co., Ltd., Shanghai, China) using a voltage range of 1.5 to $3.5 \mathrm{~V}$ vs. $\mathrm{Na}^{+} / \mathrm{Na}$. Galvanostatic charging/discharging curves and cycles were conducted using a LAND battery test system (CT2001A). All electrochemical tests were carried out at room temperature.

\section{Supporting Information}

Supporting Information is available from the Wiley Online Library or from the author.

\section{Acknowledgements}

This work was financially supported by the ERC proof-of-concept UPGREEN, ERC 2DMATER, EC under Graphene Flagship (no. CNECT-ICT-604391), the DFG via EXC1056, the National Natural Science Foundation of China (21320102006 and 51573091), and Program 


\section{WILEY-VCH}

for Eastern Scholar in Shanghai. We thank the cfaed (Center for Advancing Electronics Dresden) and the Instrumental Analysis Center of Shanghai Jiao Tong University.

Received: ((will be filled in by the editorial staff))

Revised: ((will be filled in by the editorial staff))

Published online: ((will be filled in by the editorial staff))

1. Chhowalla, M.; Shin, H. S.; Eda, G.; Li, L.-J.; Loh, K. P.; Zhang, H., Nat Chem 2013, $5(4), 263-275$.

2. Bonaccorso, F.; Colombo, L.; Yu, G.; Stoller, M.; Tozzini, V.; Ferrari, A. C.; Ruoff,

R. S.; Pellegrini, V., Science 2015, 347 (6217), 1246501-1246506.

3. Tan, C.; Zhang, H., Chem. Soc. Rev. 2015, 44 (9), 2713-2731.

4. Ciesielski, A.; Samorì, P., Adv. Mater. 2016, 28, 6030-6051.

5. Lukatskaya, M. R.; Mashtalir, O.; Ren, C. E.; Dall'Agnese, Y.; Rozier, P.; Taberna, P.

L.; Naguib, M.; Simon, P.; Barsoum, M. W.; Gogotsi, Y., Science 2013, 341 (6153), 15021505.

6. Najmaei, S.; Liu, Z.; Zhou, W.; Zou, X.; Shi, G.; Lei, S.; Yakobson, B. I.; Idrobo, J.-

C.; Ajayan, P. M.; Lou, J., Nat. Mater. 2013, 12 (8), 754-759.

7. Coleman, J. N.; Lotya, M.; O’Neill, A.; Bergin, S. D.; King, P. J.; Khan, U.; Young, K.; Gaucher, A.; De, S.; Smith, R. J., Science 2011, 331 (6017), 568-571.

8. Li, X.; Cai, W.; An, J.; Kim, S.; Nah, J.; Yang, D.; Piner, R.; Velamakanni, A.; Jung,

I.; Tutuc, E., Science 2009, 324 (5932), 1312-1314.

9. Novoselov, K. S.; Geim, A. K.; Morozov, S.; Jiang, D.; Zhang, Y.; Dubonos, S. a.;

Grigorieva, I.; Firsov, A., Science 2004, 306 (5696), 666-669.

10. Dong, R.; Pfeffermann, M.; Liang, H.; Zheng, Z.; Zhu, X.; Zhang, J.; Feng, X., Angew. Chem. Int. Ed. 2015, 54 (41), 12058-12063.

11. Kory, M. J.; Wörle, M.; Weber, T.; Payamyar, P.; van de PollStan, W.;

Dshemuchadse, J.; Trapp, N.; Schlüter, A. D., Nat Chem 2014, 6, 779-784.

12. Zhuang, X.; Mai, Y.; Wu, D.; Zhang, F.; Feng, X., Adv. Mater. 2014, 27, 403-427. 


\section{WILEY-VCH}

13. Colson, J. W.; Dichtel, W. R., Nat Chem 2013, 5 (6), 453-465.

14. Kissel, P.; Erni, R.; Schweizer, W. B.; Rossell, M. D.; King, B. T.; Bauer, T.;

Götzinger, S.; Schlüter, A. D.; Sakamoto, J., Nat Chem 2012, 4 (4), 287-291.

15. Li, X.; Wang, X.; Zhang, L.; Lee, S.; Dai, H., Science 2008, 319 (5867), 1229-1232.

16. Hernandez, Y.; Nicolosi, V.; Lotya, M.; Blighe, F. M.; Sun, Z.; De, S.; McGovern, I.;

Holland, B.; Byrne, M.; Gun'Ko, Y. K., Nature Nanotech. 2008, 3 (9), 563-568.

17. Parvez, K.; Wu, Z.-S.; Li, R.; Liu, X.; Graf, R.; Feng, X.; Müllen, K., J. Am. Chem. Soc. 2014, 136 (16), 6083-6091.

18. Yang, S.; Brüller, S.; Wu, Z.-S.; Liu, Z.; Parvez, K.; Dong, R.; Richard, F.; Samorì, P.; Feng, X.; Müllen, K., J. Am. Chem. Soc. 2015, 137 (43), 13927-13932.

19. Kim, K.; Lee, T.; Kwon, Y.; Seo, Y.; Song, J.; Park, J. K.; Lee, H.; Park, J. Y.; Ihee, H.; Cho, S. J.; Ryoo, R., Nature 2016, 535, 131-135.

20. Zou, J.; Kim, F., Nat Commun 2014, 5, 6254.

21. Zhu, C.; Han, T. Y.-J.; Duoss, E. B.; Golobic, A. M.; Kuntz, J. D.; Spadaccini, C. M.; Worsley, M. A., Nat Commun 2015, 6, 7962.

22. Li, C.; Shi, G., Nanoscale 2012, 4 (18), 5549-5563.

23. Qin, Y.; Yuan, J.; Li, J.; Chen, D.; Kong, Y.; Chu, F.; Tao, Y.; Liu, M., Adv. Mater. 2015, 27, 5171-5175.

24. Li, C.; Shi, G., Adv. Mater. 2014, 26, 3992-4012.

25. Wu, Z.-S.; Sun, Y.; Tan, Y.-Z.; Yang, S.; Feng, X.; Müllen, K., J. Am. Chem. Soc. 2012, 134 (48), 19532-19535.

26. Wu, Z.-S.; Winter, A.; Chen, L.; Sun, Y.; Turchanin, A.; Feng, X.; Müllen, K., Adv. Mater. 2012, 24 (37), 5130-5135.

27. Balogun, M.-S.; Zeng, Y.; Qiu, W.; Luo, Y.; Onasanya, A.; Olaniyi, T. K.; Tong, Y., J. Mater. Chem. A 2016. 


\section{WILEY-VCH}

28. Liu, S.; Wang, F.; Dong, R.; Zhang, T.; Zhang, J.; Zhuang, X.; Mai, Y.; Feng, X., Adv. Mater. 2016. DOI 10.1002/adma.201603036.

29. Li, L.; Wang, Y.; Pan, L.; Shi, Y.; Cheng, W.; Shi, Y.; Yu, G., Nano Lett. 2015, 15

(2), 1146-1151.

30. Pan, L.; Yu, G.; Zhai, D.; Lee, H. R.; Zhao, W.; Liu, N.; Wang, H.; Tee, B. C.-K.; Shi, Y.; Cui, Y.; Bao, Z., Proc. Natl. Acad. Sci. U. S. A. 2012, 109 (24), 9287-9292.

31. Zhao, Y.; Liu, J.; Hu, Y.; Cheng, H.; Hu, C.; Jiang, C.; Jiang, L.; Cao, A.; Qu, L., Adv. Mater. 2013, 25 (4), 591-595.

32. Zhang, J.; Zhao, Z.; Xia, Z.; Dai, L., Nat Nano 2015, 10 (5), 444-452.

33. Li, W.; Gao, F.; Wang, X.; Zhang, N.; Ma, M., Angew. Chem. Int. Ed. 2016, 55 (32), 9196-9201.

34. Qi, Y.; Mu, L.; Zhao, J.; Hu, Y. S.; Liu, H.; Dai, S., Angew. Chem. Int. Ed. 2015, 127 (34), 10049-10054.

35. Wang, Y.; Xiao, R.; Hu, Y.-S.; Avdeev, M.; Chen, L., Nat Commun 2015, 6, 6954.

36. Park, C. S.; Kim, H.; Shakoor, R. A.; Yang, E.; Lim, S. Y.; Kahraman, R.; Jung, Y.;

Choi, J. W., J. Am. Chem. Soc. 2013, 135 (7), 2787-2792.

37. Yu, G.; Jie, K.; Huang, F., Chem. Rev. 2015, 115 (15), 7240-7303.

38. Shimizu, T.; Masuda, M.; Minamikawa, H., Chem. Rev. 2005, 105 (4), 1401-1444.

39. Liu, S.; Zhang, J.; Dong, R.; Gordiichuk, P.; Zhang, T.; Zhuang, X.; Mai, Y.; Liu, F.; Herrmann, A.; Feng, X., Angew. Chem. Int. Ed. 2016, 55, 12516-12521.

40. Liu, S.; Gordiichuk, P.; Wu, Z.-S.; Liu, Z.; Wei, W.; Wagner, M.; Mohamed-Noriega, N.; Wu, D.; Mai, Y.; Herrmann, A.; Müllen, K.; Feng, X., Nat Commun 2015, 6, 8817.

41. Nguyen Thi Le, H.; Bernard, M. C.; Garcia-Renaud, B.; Deslouis, C., Synth. Met. 2004, 140 (2-3), 287-293.

42. Wang, J.; Ober, C. K., Macromolecules 1997, 30 (24), 7560-7567. 


\section{WILEY-VCH}

43. Yang, S.; Feng, X.; Wang, L.; Tang, K.; Maier, J.; Müllen, K., Angew. Chem. Int. Ed. 2010, 49 (28), 4795-4799.

44. Zhu, L.; Shen, Y.; Sun, M.; Qian, J.; Cao, Y.; Ai, X.; Yang, H., Chem. Commun. 2013, 49 (97), 11370-11372.

45. Su, D.; Zhang, J.; Dou, S.; Wang, G., Chem. Commun. 2015, 51 (89), 16092-16095.

46. Zhou, M.; Li, W.; Gu, T.; Wang, K.; Cheng, S.; Jiang, K., Chem. Commun. 2015, 51 (76), 14354-14356.

47. Chen, X.; Liu, L.; Yan, Z.; Huang, Z.; Zhou, Q.; Guo, G.; Wang, X., RSC Adv. 2016, 6 (3), 2345-2351.

48. Xiang, X.; Zhang, K.; Chen, J., Adv. Mater. 2015, 27 (36), 5343-5364.

49. Yabuuchi, N.; Kubota, K.; Dahbi, M.; Komaba, S., Chem. Rev. 2014, 114 (23), 1163611682.

50. Wang, X.; Kajiyama, S.; Iinuma, H.; Hosono, E.; Oro, S.; Moriguchi, I.; Okubo, M.; Yamada, A., Nat Commun 2015, 6, 6544. 


\section{WILEY-VCH}

We demonstrate a bottom-up approach towards 3D hierarchical macroporous polypyrrole aerogels via soft template-directed synthesis and self-assembly of ultrathin polypyrrole nanosheets in solution, which present the interconnected macropores, ultrathin wall and large specific surface areas, thereby exhibiting a high capacity, satisfactory rate capability and excellent cycling stability for $\mathrm{Na}$-ion storage.

Keyword: three-dimensional (3D) $\bullet$ macroporous $\bullet$ self-assembly $\bullet$ conducting polymer $\bullet$ nanosheets

Shaohua Liu, Faxing Wang, Renhao Dong, Tao Zhang, Jian Zhang, Zhikun Zheng, Yiyong Mai, and Xinliang Feng*

Soft-Template Construction of 3D Macroporous Polypyrrole Scaffolds

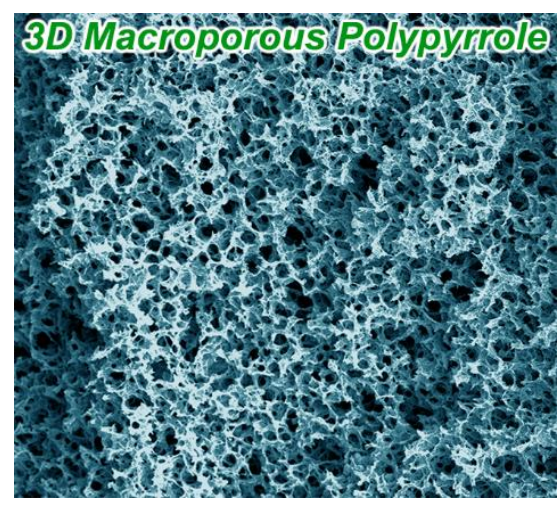

